\title{
El Libro del arte de cozina de Diego Granado Maldonado. Estado del arte
}

\author{
Diego Granado Maldonado's Libro del arte de cozina
}

\author{
NANCY DE BENEDETTO \\ alfonsina.debenedetto@uniba.it
}

Università degli Studi di Bari

\begin{abstract}
Resumen: Sobre el recetario de Diego Granado no se ha escrito mucho y siguen circulando hipótesis y errores que dependen básicamente de la falta de una edición crítica que aclare la procedencia de las diversas partes del texto y, sobre todo, que facilite su lectura. Se trata de un texto muy heterogéneo y algo caótico con el que me enfrento aquí solo para presentar un análisis de las cuestiones más importantes relativas al tratamiento de sus dos fuentes principales. Por una parte, realiza Granado un plagio del Libro de guisados de Robert de Nola (1529). Por el otro, traduce buena parte de la Opera de Bartolomeo Scappi (1570). El método de trabajo del autor revela diversas incongruencias cultuales y lingüísticas que se han observado respecto al tratamiento de los ictiónimos.
\end{abstract}

Palabras clave: tratados, recetarios, textos fuente, traducción, ictiónimos.

Abstract: Not much has been written about diego Granado's cookbool and hypotheses and errors continue to circulate that basically depend on the lack of a criticale edition that clarifies the origin of the various parts of the text and, above all, that facilitates its reading. It is a very heterogeneous and somewhat chaotic text wchich I am faced here only to present a recognition of the most important questions regarding the treatment of its two main sources. On the one hand Granado plagiarizes Robert de Nola's Libro de guisados (1529) which edition we fix here. On the other he tranalates the main part of Bartolomeo Scappi's Opera (1570). The author's working method reveals various cultural and linguistic inconsistencies that have been observed in relation to the treatment of ichthionyms.

Keywords: treaties, cookbooks, source texts, translation, ichtionyms. 
Sobre el recetario de Diego Granado no se ha escrito mucho y siguen circulando hipótesis y errores que dependen básicamente de la falta de una edición crítica que aclare la procedencia de las diversas partes del texto y, sobre todo, que facilite su lectura. Se trata de un texto muy heterogéneo y algo caótico con el que me enfrento aquí solo para presentar un análisis de las cuestiones más importantes relativas al tratamiento de sus dos fuentes principales.

Diego Granado publicó el Libro de arte de cocina en 1599, en Madrid (por Luis Sánchez), apoderándose literalmente, sin citarlos, por un lado, del Libro del mestre Robert de Nola, el viejo Libre del coc (1520), que copió de una de las diversas ediciones castellanas que se imprimieron a lo largo del siglo XVI, y por el otro de un enorme corpus de recetas prelevado y traducido de la Opera de Bartolomeo Scappi, cocinero privado del papa Pío V (1570). De un último grupo, por último, se desconoce la procedencia, a no ser que se quiera admitir que quizás formen parte de un recetario particular del autor, del que, sin embargo, seguimos teniendo escasísimas noticias biográficas (Allard Dromer: https://dbe.rah.es/biografias/97563/diego-granado-maldonado). Hasta tiempos relativamente recientes -los años 90 del siglo pasado- se conocía realmente muy poco el Arte de cozina de Granado ya que circulaba solo en una trascripción hecha por Joaquín del Val para la Sociedad de bibliófilos españoles, en 1971. Se suponía que procedía de recetarios anteriores, pero, a pesar de que ya Joaquín del Val había tenido en cuenta el plagio del Libro de Cozina del maestro Robert (1971: XXIII), se le seguía considerando original en contribuciones muy acreditadas sobre filología y gastronomía (Pérez Samper 1992) y se le atribuían rasgos de genialidad culinaria que en realidad correspondían a otros grandes cocineros, como demostró finalmente Gloria Guidotti (1996), al llevar a cabo un primer y acertado control filológico.

En el título completo de la princeps, ${ }^{1}$ Granado expresa aspiraciones muy ambiciosas además de un buen conocimiento de los materiales que tenían que aparecer en un libro de cocina, aunque no podía haber acudido a ninguna tradición española, ya que casi no había antecedentes que tuvieran características parecidas:

Libro del arte de cozina, en el qual / se contiene el modo / de guisar de comer en cualquier / tiempo, assi de carne como de pescado, para sa-/nos y enfermos, y convalecientes, assi de / pasteles, tortas, y salsas, como de con- / servas a la usanza Española, Italia- / na y Tudesca de nuestros tiempos (G 1609).

El título, en realidad, presenta unos contenidos ya clásicos en los tratados gastronómicos europeos -sobre todo italianos- al contener manjares y potajes de cualquier tiempo (Cuaresma y otros diversas estaciones del año), cocina para dolientes, pastelería y salsas, o sea mixturas de especias,

1 A parte la primera edición, el Libro de arte de cozina se imprimió solo dos veces más: en Madrid, 1609, por Alonso Martín, y en Lleida en 1614, por Luys Manescal. En tiempos más recientes, se hizo una edición del texto de 1614 a cargo de la Diputació de Lleida en 1991, según criterios más celebrativos que filológicos. La edición de referencia en el presente trabajo es la de Madrid 1609, que en las citas se indicará como G, ya que es disponible en línea. 
la secuencia del banquete, etc. La promesa de internacionalidad contenida en el título se da por explicada, debido a la procedencia heterogénea del libro. Respecto a las «usanças españolas», el texto es muy contradictorio porque, por un lado, Granado demuestra tener buenos conocimientos regionales, al parecer sobre todo levantinos; pero, por otro, debido al hecho de tratarse también de la traducción de un texto italiano, presenta un grado de exotismo tan elevado que se infiere que no tenía conocimiento directo de muchos alimentos y recetas totalmente desconocidos en España. En definitiva, el Arte de cozina es una operación que procedía del mundo de los libros, más que del de las cocinas. En este sentido, hay que señalar que la mención a la gastronomía «tudesca», de la que presume Granado en el título, no tiene mucho que ver directamente con el mundo germánico, puesto que se refiere solo a un modo de guisar «a la tudesca» que formaba parte de técnicas y recetas conocidas ya desde el siglo Xv, como a la catalana por diversas recetas de sopas de calabaza, a la italiana por recetas en las que se sustituía la leche de almendras con aceite de oliva, a la veneciana, etcétera. Las recetas en cuestión, en realidad, se limitan a dos, ambas sacadas de Scappi: Para ahogar el lomillo de vaca a la tudesca (G: 40) y Para guisar Truchas (G: 168 v).

En el frontispicio aparece, por debajo del título que hemos copiado arriba, una segunda descripción que, confiriendo cierta simetría a la materia de que trata el libro, está dedicada a

Los officios necessa/rios que comunmente hay en la casa de los señores, $\mathrm{pa}-/ \mathrm{ra}$ el regimiento de la casa de los Grandes, y Cava-/lleros:el principal de los quales es el Mayordomo,/y después Camarero, y Guardaropa, Maestresala,/Copero,Trinchante, Cavallerizo,/Veedor,Despensero,/Cozinero (G: fol. 1).

Los oficios remiten al Libro de guisados del maetro Robert, ya que la descripción está copiada de la segunda edición castellana de Logroño de 1529. A pesar del orden del frontispicio, las dos partes están invertidas en la dispositio de Granado, ya que en su recetario aparecen la parte procedente de Nola primero, y luego el corpus sacado de Scappi.

\section{De los tratados al recetario}

Granado se nos presenta desde un principio por su primera y esencial característica de libro híbrido, según una perspectiva tanto cultural como lingüística. Primero juntó dos textos lejanos, pero que cada uno, históricamente, habían expresado las excelencias del ars coquinaria en su momento, ya que procedían ambos del ambiente cortesano. El tratado de Nola tiene un largo historial y proviene de un antiguo libro catalán que fue escrito en la segunda parte del siglo Xv y que con razón puede ser considerado el último gran libro medieval de área mediterránea (Santanach 2020). El hecho de que se difundiera algunas décadas después, ya en época renacentista, a partir tanto de la princeps catalana (Barcelona 1520) como de la castellana (Toledo 1525), dio lugar a hipótesis y deducciones erróneas sobre una edición incunable de 1477 que en realidad nunca existió, aunque sí es cierto que el tratado circulaba manuscrito ya antes de esta fecha (Santanach 2020: 93). Este tratado tuvo 
una larguísima difusión, puesto que se imprimió siete veces en catalán y por lo menos diez en castellano, a medida que sus rasgos se modificaban parcialmente añadiéndosele recetas y capítulos que pretendían convertirlo en un libro de facciones modernas respecto a la princeps. Para no alejarnos del tema de este estudio, nos ceñimos a la tradición castellana, mencionando las ediciones que nos serán útiles a la hora de llevar a cabo una comparación con Granado. Se trata de la ya mencionada princeps de Toledo 1525, conocida con el título abreviado Libro de cożina, que lleva algunas diferencias con la princeps catalana, ya que contiene cerca de 20 recetas más, además de una agrupación parcialmente distinta. La edición de Logroño de 1529 contiene, en cambio, por primera vez una justificación que se amolda a la tópica editorial de la época donde se especifica que el tratado se habría hecho tras la petición directa del soberano a su cocinero, Robert (Cruz Cruz: 228-229). Esta, que es conocida como Libro de guisados, es la edición más completa, ya que agrega algunas recetas nuevas respecto a 1525 y añade el segundo capítulo sobre «Doctrina saludable para la gobernación de las casas de los caballeros y personas de mediano estado [...]» (Cruz Cruz 1997: 255-261). A través de sus nuevas ediciones, la obra del maestro Robert iba acentuando unas características que la adaptaban al tratado didáctico moderno y abierto a un público más extenso. Es muy difícil que las enmiedas de 1529 procedieran de la mano del mismo autor, debido a una cronología que se hace demasiado larga respecto a la primera redacción de la obra, mientras que es más probable que se ocupara de ello el mismo redactor que trabajó en el texto de Toledo 1525 (Cruz Cruz: 19).

La publicación de la Opera de Bartolomeo Scappi se coloca en una tradición que contaba ya más de un siglo y que remitía seguramente a una tradición francesa más antigua aún. A mediados del XVI lucía ilustres exponentes, como Messisbugo, Panunto, Rossetti que describieron en sus tratados las costumbres del espacio mítico de la corte bajo una perspectiva de alta especialización (Lefèvre 2003). Y, de hecho, a partir de los Banchetti de Cristoforo Messisbugo, el tratado de cocina se convirtió en género literario. La Opera de Scappi se impuso por ser «certamente la più completa fra tutte le edizioni di arte culinaria del Cinquecento» puesto que además de un corpus de 1017 recetas (Di Schino-Luccichenti: 55) contenía la descripción de la cocina como espacio arquitectónico complejo, el esquema fijo de la organización del banchetto, según el patrón de Messisbugo, o la formación de la alta profesionalidad del cocinero de corte, entre otros elementos. La Opera también, a la par del libro de Nola, tuvo mucho éxito y, sobre todo, fue un libro fundamental del Renacimiento italiano más allá de los límites del libro de cocina para «diventare una vera e propria testimonianza storico culturale sul costume della corte rinascimentale» (Di Schino-Luccichenti: 56).

En España, antes del libro de Granado se habían imprimido solo el Arte cisoria del marqués de Villena, un tratado que nombra muchos alimentos pero que no contiene recetas, y el libro del maestro Robert, o sea no existia una tradición parecida a la italiana ni a la francesa (Allard 1997). En efecto, al publicar Granado su Arte de cozina, en Madrid, no quiso hacer un tratado de gastronomía sino un recetario y por ello fue recortando de sus fuentes las partes más didascálicas y didácticas que la ascribían al género tratado, tanto del libro de Nola, como de la Opera. 


\section{Corpus}

El Arte de cozina de Granado procede, pues, de dos libros de ambiente cortesano, que representan una cocina básicamente medieval, la del mestre Robert, y otra proyectada en un riquísimo presente renacentista. La unión no deja de presentar, en primera instancia, un hibridismo cultural posiblemente homogeneizado solo por pertenecer ambas fuentes a un mundo gastronómico precolombino en el que no se habían difundido aún alimentos muy importantes de origen americano y en el que, sin embargo, todavía se hacía uso de gran cantidad de especias, azúcar y agua de rosas, aunque en medidas muy inferiores en el tratado italiano que en el catalán. No cabe decir que los conocimientos sobre los alimentos que muestra Scappi, así como sus mismas recetas son mucho más complejas y pormenorizadas que las de Nola, y, de hecho, también lingüísticamente, el recetario de Granado presenta profundas disparidades a partir del carácter híbrido de la operación misma de su refundición, puesto que compagina una transcipción y una traducción. Las dos fuentes de Granado presentan un corpus muy distinto, ágil y conciso el del Mestre Robert, en el que se suman 242 recetas; enorme, didascálico, ordenado y vario el de Scappi, que asciende a más de 1000. El Arte de cozina se compone por la yuxtaposición de la primera parte del tratado de Nola, más un corpus importante de recetas sacadas de Scappi a las que se añaden un tercer corpus de momento anónimo. Ya que no es especial objetivo de este estudio hacer una comparación precisa, y en términos generales, concordamos por lo que se refiere al corpus sacado de Nola, nos quedamos con la recognición que hizo Jeanne Allard Dromer:

De hecho, de las setecientas sesenta y dos recetas que ofrece la obra de Granado, quinientas ochenta y siete provienen de la Opera de Scappi, cincuenta del Libro de Cozina de Ruperto de Nola, quedando ciento veinticinco sin poder identificarse su procedencia (https://dbe.rah. es/biografias/97563/diego-granado-maldonado).

Por lo que se refiere a la disposición, Granado realiza una inversión de los materiales procedentes del maestro Robert, que empezaba con los cortes y seguía con los oficios. Su Arte de Cozina se abre, sin embargo, con nueve párrafos sobre los oficios tal como aparecían dispuestos por Nola que no incluyen el décimo, el caballerizo, aunque aparece en el frontispicio que hemos reproducido arriba. Siguen luego tres capítulos - X-XII — sobre aguzar cuchillos, dar agua para lavar las manos, poner la mesa; de estos capítulos que figuraban entre los cortes y los oficios, falta en Granado el dedicado al Dar a beber, que comprendía la larga ceremonia de la salva. Los 13 párrafos dedicados a los cortes de las carnes y de las aves aparecen todos en su orden original. Luego empieza un corpus de unas cuarenta y dos recetas tomadas de Nola que termina con la Treballa que se dice salsa de ansarones (G: 36 r) a las que siguen la inmensa serie seleccionada de la Opera de Scappi, pero hay que añadir que unas 8 más de Nola que están mezcladas a lo largo del texto y a las que a veces Granado cambia el título. Para terminar, hay que calcular que hay un tercer corpus de recetas, mezcladas con las diversas partes sacadas de Scappi (más de 120 según alega Allard) de las que no se conoce la fuente y que por lo visto lingüísticamente se parecen más a modelos ibéricos —entre Nola y Montiño—, que italianos. 


\section{Textos fuente}

Según trasmiten los estudios que se han hecho sobre el tema, la edición del tratado de Nola que Diego Granado refundió en su recetario es el Libro de cocina de 1525 (Cruz Cruz: 21), o sea la princeps castellana de Toledo (a partir de ahora T), ${ }^{2}$ pero esta derivación no es segura y acerca de este asunto se ha acumulado mucha confusión (Von Gamminen: 407). Personalmente, he podido comprobar que diversas recetas remiten al Libro de guisados, o sea a la edición de Logroño de 1529 (a partir de ahora L). ${ }^{3}$ Más allá de numerosos cambios mínimos que no podemos registrar aquí, me limito a señalar, por ejemplo, que El Relleno de cabrito es una receta mucho más larga en T (65) que en L (284r) y en Granado (12 v) así como el Buen broete con caldo de carne que se dice esponja tiene una línea más en T (74) que en L (30 v) y en Granado (16 r). Luego hay dos recetas que llevan la marca «añadido» $\mathrm{O}$ «emendado», precisamente porque en $\mathrm{T}$ eran diversas o no estaban, tal como confirma Cruz Cruz en su edición de L de 1997 (pp. 264-265 y 306-307). Se trata de Las especias de hipocras añadido y la Pólvora de duque emendado tomadas de la enumeración de salsas y especias que abren el listado de recetas tomadas de Nola y que, sin embargo no aparecen en su orden original (G: $11 \mathrm{r}$ ). A estas dos, se suman tres más, colocadas en el Tablero de cocina para convalecientes y enfermos (G: 288 r), que, de la misma manera, se añadieron al texto de L: Almendras [sic] para dolientes (G: 314 r), Otra almendrada para dolientes muy debilitados (G:314 v), Otra almendrada para dolientes que tienen gran calenturay grandes ardores (Granado: $314 v$ ). Cinco recetas nuevas, pues, más las variantes del Relleno de cabrito y del Buen broete con caldo de carne que se dice esponja, nos parecen suficientes para afirmar que no hay dudas sobre el hecho que la edición de la que deriva Granado su relación con Nola es L. Recordemos, además que ya la segunda parte del frontispicio, relativa a los oficios, está sacada de L, como se ha evidenciado arriba.

La ortografía del texto de Granado se ha modernizado respecto al texto del maestro Robert, como es obvio, puesto que entre los dos hay un desfase editorial de por lo menos 70 años. Los rasgos más modernos se encuentran en el sistema de puntuación donde los antiguos dos puntos polifuncionales se sustituyen mayoritariamente per comas; así como también se observan en la la morfología: el verbo haber que se escribe ahora con $h$, y a la explicitación de las nasales.

Con respecto a la edición de Scappi que Granado tuvo entre las manos, la cuestión no presenta problemas porque el texto fuente no sufre variaciones y porque seguimos disponiendo de hipótesis muy vagas sobre los contactos históricos que pudo mantener Granado con Italia y con el libro del cocinero secreto de los papas.

En principio se distinguen las recetas sacadas de las dos fuentes por diversos elementos de estilo y lengua, entre los que figuran en una mínima parte el título, que suele ser nominal en el caso del

2Como texto de referencia remito a la edición de Carmen Iranzo de 1969.

3 Ya que la edición de Cruz Cruz (1997) no evidencia todas estas variantes, he tomado como referencia L, disponible en línea. 
mestre Robert, mientras que a menudo es perifrástico en Scappi. Granado confirma la diversidad de los originales y al lado de recetas como Salsa de ansarones, broete de madama, o busaje de conejo pone recetas como Para hacer pastel de conejos caseros, o Para hacer tortitas de yervas a la Lombarda. Sin embargo, esta diferencia se realiza en muchos casos aunque no siempre, porque por lo general Granado simplifica y tiende a nominalizar también los títulos de Scappi, que en el texto fuente eran todos perífrasticos, según un modelo estilístico más o menos fijo en los tratados italianos del siglo XvI. Así que, mientras el título solo puede ser un elemento secundario en la distinción de las fuentes, más relevante resulta el incipit de las recetas que se relaciona con el conjunto textual de manera más clara. Se abren con infinitivos con función de imperativo, por ej. tomar, majar, pasar o tomar as, / has de tomar, las recetas sacadas de Nola, mientras que lo hacen con imperativos aglutinados como tómese/tómense, bátase/bátanse, úntese, límpiese, pélense, etc, las recetas traducidas del italiano (Guidotti: 223). A los dos tipos de íncipit corresponde una organización sintáctica sintética y coordinativa, que es la de Nola, más descriptiva y tal vez compleja la que procede de Scappi, calcada sobre el patrón de receta creada por Cristoforo Messisbugo que tendrá vigencia a lo largo de todo el siglo XVII en Italia. Las dos tipologías, aunque en presencia de esporádicas manipulaciones de los originales, resultan muy bien reconocibles, como en los ejemplos que traemos aquí:

Capirotada de perdices

Tomar perdices y pelarlas, y ponerlas entre las brasas o rescoldo, y que hayan estado un pensamiento, sacarlas y limpiarlas, y assarlas, y lardearlas bien, y después de assadas, cortarlas como para hacer platos dellas, y después rallar un buen queso anexo, y tomar dos cabeças de ajos assados, y mondarlos muy limpiamente, y majarlos, y después poner el queso en el mortero ... (Granado 15 r).

Para hacer pisto de carne de ternera.

Tomense las pulpas de la carne de la ternera aquellas que estan debaxo de la espalda, y los lomillos, y haganse mas de medio cozer, con que sea la ternera muerta del proprio dia, porque sea mas sustanciosa, majense en el mortero con un migajón de pan tostado o por tostar, y en estando bien majado destemplarse ha con caldo magro de la misma ternera, y passarse ha por el cedaço, por el colador, y cuezase como los demas (Granado, 296 v).

Por lo que concierne al corpus no atribuido, no tengo la posibilidad de aportar elementos nuevos, ante todo porque no se ha hecho aún un estudio crítico, que me conste, ni siquiera a nivel de análisis comparativo de los títulos. Lo único que me puedo atrever a reseñar aquí es que el último capítulo de Granado, Para hacer diversas maneras de conservas, contiene muchas recetas que no he encontrado en ninguna de las dos fuentes conocidas. Se trata de confitería y frutas en almíbar, que no parece tener familiaridad con la organización sintáctica de las recetas de Scappi, como traemos a continuación:

Rosa confitada:

Tomar açucar muy blanco y cozerlo hasta que este subido muy frio: y tener la rosa fina deshojada, y 
echarla dentro, y tenerla dentro un rato, yendola rodeando, y luego ponerla a cozer a fuego manso, rodeandola muy a priessa, porque se embueve el açucar, y echarle almizcle, y en estando seco, echarlo en su cedaço, y abrirle la hoja cada una de por si y cernerla de açucar (Granado 323 r).

\section{Notas sobre la traducción}

Por lo que atañe a la enorme parte traducida del tratado de Scappi, emerge una gran abundancia de calcos, préstamos y neologismos que conllevan un grado de exotismo muy elevado, por lo que se sospecha muy plausiblemente que Granado no conociera muchos de los alimentos y las técnicas que formaban parte del enciclopédico patrimonio al uso del cocinero de Pío V. Granado, de hecho, importa en su traducción una exorbitante serie de culturemas que aparecían en los hiperepecializados conocimientos regionales de Bartolomeo Scappi; esos elementos pertenecen propiamente a las categorías de los denominados imposibles en la teoría de la traducción, precisamente porque no existen equivalentes en la lengua de llegada. Pues, Granado, por un lado, neutraliza los localismos, como en el caso de las mandolas ambrosinas, que aparecen en muchísimas recetas de Scappi, y que se convierten en almendras genéricas, así como el riso de Salerno lo hace en arroz sin denominación de origen. Por otro lado, y según una tendencia opuesta y prevalente, pretende remitir a productos y procedencias tan remotas y pormenorizadas que provocan un difuso efecto de opacidad. Además, intenta presentar de manera contrastiva usos y tratamientos de algunos alimentos en las costumbres italianas y españolas o valencianas, como en el caso de la receta de la Escudilla de arroz con leche de almendras, donde no deja de añadir que «en Valencia se hace con tanta curiosidad, que va cada grano por sì (Von Gemmingen, p. 415). Por lo contrario, resulta muy curioso que copie, sin aportar intervenciones propias en la preparación ni informaciones regionales, la receta de la olla podrida, donde recorta, sin embargo, las explicaciones que proporciona Scappi cuando afirma que «si nomina dalli Spagnoli in questo modo, percioche per la maggior parte si fa in pignate di terra, le quali dimandano oglias, et potride chiamano diverse materie ben cotte» (65). Evidentemente tales explicaciones hubieran resultado absurdas en un texto imprimido en Madrid.

Respecto a los pescados, debido a la pretensión de reproducir la enorme riqueza del capítulo III del tratado de Scappi, gran parte de ellos resultan irreales y desconocidos en la España del siglo XVI, aunque se consumía mucho pescado ya en la época medieval por la observancia cuaresmal (Domingo 2003: 25). A pesar de ello, las variedades conocidas en los mercados de Madrid eran pocas (Allard Dromer: https://dbe.rah.es/biografias/97563/diego-granado-maldonado), como resulta también en el primer tratado cortesano castellano, el Arte de cocina de Martínez Montiño (1611), en el que he contado solo 16 variedades, a parte los mariscos y la descripción del estocafix (Montiño: 504). En el Libro de Guisados de Nola, que es de área mediterránea y no castellana, el número de pescados es relativamente más alto, ya que aparecen 22 variedades que se elaboran básica y monótonamente de tres maneras: en pan, en cazuela y cocidos. La variedad de Granado es muy elevada, en cambio, puesto que procede del inmenso caudal de Scappi, que solo al sollo, por ejemplo, dedica 24 recetas. Cabe recordar que la Opera, precisamente, se diferencia de todos los tratados contemporáneos por el espacio que dedica al pescado, alimento valorizado en Italia especialmente en el Renacimiento, respecto a las épocas anteriores (Di Schino- 
Luccichenti, p. 63). Por el contrario, cabe destacar que en España Montiño todavía dedicará poquísimo espacio al tema por ser los pescados alimentos que no puedan demostrar la ciencia y la capacidad del cocinero, por lo cual admite de manera explícita el autor: «Trataré un poco de pescados aunque no me pienso detener mucho en ello, porque los pescados, quanto toca à conocimientos, y frituras, que son las dos maneras ordinarias, casi van todos por un camino (...)» (270).

En Granado, aparecen por lo menos 52 variedades diversas de pescados, que es una cifra que puedo asegurar por los nombres que aparecen en los títulos de los apartados descriptivos y de las recetas, aunque dezconozco si hay otros que se puedan encontrar en el corpus de otras preparaciones.

Por lo que concierne al comportamiento traductivo, errores de interpretación que remiten a localismos italianos han sido analizados por Gloria Guidotti (1996) que incluso señala cortes y facilitaciones que tienden a anular la eficacia de la labor de Granado por incompetencia lingüística y falta de conocimientos directos de los alimentos. Atención particular se ha dedicado a las recetas y a la descripciones de ictiónimos, por lo que vamos ahora a profundizar algunos aspectos. En este ámbito, hay que señalar una notable tendencia a la transcripción fonética en casos como el pez Sampietro, el pez iñudo (it. Ignudo), el fragolino, y al neologismo en casos con muchas ocurrencias como escórfeno, gongola, embrina, esgombro; se trata de nombres que no existen en castellano, y no se en cuentran registrados en ninguno de los diccionarios históricos del repertorio del NTLLE de la Real Academia. Las descripciones de los pescados, como la mención de las costas de su procedencia según las temporadas del año, proceden de Scappi y resultan del todo ajenas a las variedades regionales españolas. Véase en propósito, para poner algún ejemplo, el pescado que en «Venecia les llaman Cefali de buen budello en la valle de Comarechio (sic por Comacchio), letragano. En la Toscana magini» (Scappi: 65 r). Otro caso muy significativo y por esto ya citado por otros estudios, el del robalo que en Italia llaman espigolo, en Venecia varoli, en Génova lupi, en Florencia y Pisa ragni y que «quando entran del mar en el rio son perfetissimos, y en el Tiber, y en el Po, son blanquissimos plateados» (Von Gemminen: 405).

A pesar de ello, a parte estas características de inaceptabilidad de la traducción, según la definición de Gideon Toury (2004: 198), me interesa aportar unas reflexiones sobre el tratamiento de ictiónimos muy comunes en Italia y en España, tanto en las costas de área mediterránea como en las de la atlántica. Se trata de casos de pescados que tienen relevancia en el recetario de Granado puesto que aparecen en muchas ocurrencias. Fijémonos en la descripción del «esgombro» que Granado deriva refundiendo dos capítulos de Scappi (117-118), y poniéndole el título Del pescado llamado en Italia Esgombro y en Francia Maccario (G: 191 v), que, por muy aclarecedor que pretendiera parecer, resulta, en cambio, muy ópaco, ya que ninguno de los dos nombres era conocidos en España. Por supuesto, el hecho de que no lo asociara a la caballa, revela que posiblemente no lo conocía o, por lo menos, que no sabía que se trataba de la misma variedad. Porque, sin duda, no deja de ser muy extraño que dedique otro apartado, aparentemente original, al Pescado llamado Disoly en Castilla Cavalla (G: 150 v), que no parece tener mucha relación con el 'esgombro' y con la descripción que de él proporciona Scappi. Algo parecido pasa con el pez triglia que Granado traduce con «pescado llamado en Italia Tiglia, y en España Besugo, y en algunas playas Pagel», engañadose quizás por el color que, según 
la descripción de Scappi, tiene la de mejor calidad «è rossa quanto il foco» (Scappi 116), «roxa encendida», pero que en realidad ninguna característica comparte con el besugo ya que «tiene la cabeza corbada como el puerco» y «dos pendientes en la barba» (G: 151r). En la descripción Del pescado llamado en Italia Sampietro mantiene que en España al mismo pescado se le llama «sardina» aun siendo «corpulento», y a pesar de que «guisase de todas las maneras que el rodavallo» (G. 194 r).

La impresión que se deduce es que, por lo general, Granado traducía sin tener al alcance herramientas lingüísticas específicas, aunque, de hecho, en aquella época realmente podía contar solo con el diccionario de Las Casas, donde aparecen muy pocas especies de ictiónimos frente a la gran cantidad que presenta en su Arte de Cocina. Muchos de ellos son registrados por diccionarios muy posteriores a 1599 y, por lo general, la codificación además, como sabemos, es mucho más tardía e ineficaz respecto al uso del léxico material, por lo cual no es del todo reveladora de su difusión, aunque nos proporcione orientaciones diacrónicas importantes. ${ }^{4}$ En cuanto a algunos pescados comunes, los diccionarios recogidos en el NTLLE registran muy precozmente tanto «besugo» como «caballa» que, sin embargo, no aparecen en Las Casas, mientras que precisamente la triglia en este se traduce con salmonete (147), como en nuestros días, y el pez sgombro se traduce con «aleche» (131). El céfalo, que aparece en numerosas ocurrencias en el Arte de cocina, en realidad no se registra nunca en los diccionarios históricos, mientras se traduce en Las Casas como «albur», voz que encontramos, en cambio, en todas las herramientas recogidas en el NTLLE, a partir de Nebrija (1495) y que es sinónimo de mújul. El hecho de que Granado prefiriera el calco incluso en estos casos de términos tan corrientes nos proporciona la calidad de un método de trabajo muy aleatorio, que si no se basa en investigaciones lingüísticas siquiera esenciales, tampoco lo hace en el conocimiento directo de muchas de las especies que trata.

\section{Conclusiones}

El análisis que he llevado a cabo evidencia que Granado no era un lingüista ni un literato, como en efecto, ya sabíamos. Si buscamos una comparación en cierto sentido opuesta, se me ocurre el tratado del marqués de Villena, que en El arte de cortar con cuchillo que, además de enumerar por lo menos 43 pescados distintos y 4 nombres de mariscos, demuestra conocerlos todos, ya que en ningún momento aparecen gongolas en lugar de almejas, ni besugos que se confundan con sardinas (126-127). De la misma manera, surgen dudas sobre la propia formación de Granado y su perfil de cocinero. A pesar de ello, las posibilidades de investigación que ofrece su recetario son interesantes, tanto bajo la perspectiva de estudios comparativos sobre el léxico de las profesiones, como desde la expectativa de estudios culturales sobre los distintos usos gastronómicos que entran en contacto en su Arte de cozina.

4Especialmente sobre la dificultad de la descripción y la heterogeneidad de sistematización de los pescados, véase Mondejar 1999. 


\section{Bibliografía}

Allard, J. (1988) «La cuisine espagnole au siècle d'On», Mélanges de la Casa de Velárquez, XXIV, pp. 177-190.

Allard Dromer, J. «Granado Maldonado Diego», Diccionario Biográfico de la Real Academia de la historia (https://dbe.rah.es/biografias/97563/diego-granado-maldonado).

Aragón, E. Marqués de Villena (1766 [1a ed. 1423]) Arte cisoria, ó tratado del arte de cortar del cuchillo que escrivió Don Henrique de Aragón marqués de Villena [...]. Madrid, Oficina de Antonio Marin.

Benet i Pinós, X. (1961) «Justificación de la edición». Libro del arte de cocina, Lleida, Pagès.

Catricalá, M. (1982) «La lingua dei Banchetti di Cristoforo Messisbugo», Studi di lessicografia italiana, IV, 1982, 147-268.

Cruz Cruz, J. (1997) La cocina mediterranea en el inicio Renacimiento. Martino da Como "Libro de arte culinaria". Ruperto de Nola "Libro de guisados", Huesca, La Val de Onsera.

Di Schino, J., Luccichenti, F. (2007) Il cuoco segreto dei papi, Roma, Gangemi Editore.

Gemminen, B. von. (1995) «Estudios lexicológicos sobre la lengua culinaria del Siglo de Oro, Revue de Linguistique romane, 59, pp. 401-417.

Lefèvre, M. (2003) «Res culinaria e Ars coquinaria: distinzioni, analisi di genere ed esperienze specifiche nell'ambito dei trattati di cucina del Cinquecento», en C. Spila (ed.) La sapida eloquenza. Retorica del cibo e cibo retorico, Roma, Bulzoni, pp. 113-133.

Granado Maldonado, D. (1609) Libro del arte de cozina : en el qual se contiene el modo de guisar de comer en qualquier tiempo, assi de carne como de pescado, para sanos y enfermos y conualecientes, assi de pasteles, tortas y salsas como de conseruas a la vsança española, italiana y tudesca de nuestros tiempos / porDiego Granado, Madrid, Alonso Martín. [1599] chttp://www.bibgirona.cat/pandora/viewer.vm?id=0000000172)

Guidotti, G. (1996) «La gastronomía come percorso differenziale di cultura: il percorso Scappi-Granado», Cuadernos de filología italiana, 3, Madrid, Servicio de publicaciones de la UCM, pp. 221-237.

Martínez Montiño, F. (2006) Arte de cocina, pastelería vizcochería, y conservería, Valladolid, Maxtor. Facsímil de Barcelona 1763.

Mondéjar, J. (1999), «Onomasiología ictionímica y diccionario de la lengua (cuestiones metodológicas y prácticas)», Anuario de Estudios Filológicos, XXII, 301-318.

Nola, R. (1997 [1a ed. 1529]) Libro de guisados, manjares y potajes intitulado Libro o de cocina, Juan Cruz Cruz ed., pp. 227-395.

Nola, R. (1969 [1a ed. 1525]) Libro de cozina, Carmen Iranzo ed., Madrid, Taurus Ediciones.

Nola, R. (2018 [1a ed. 1529]), Libro de guisados, manjares y potajes intitulado Libro de cozina, Logroño, Miguel de Erguía. En Raccolta di testi per la storia della gastronomia, digitalizado por Edoardo Mori (https://www.mori.bz.it/gastronomia/Rupert $\% 20 \mathrm{da} \% 20$ Nola $\% 20 \% 20 \mathrm{Libro} \% 20 \mathrm{de} \% 20$ Guisados.pdf) 
Nancy De Benedetto. El Libro del arte de cozina de Diego Granado Maldonado. Estado del arte

Pérez Samper, M. A. (1992) «Los recetarios de cocina (siglos XV-XVII)», en Codici del Gusto, Maria Grazia Profeti, ed., Verona, Franco Angeli, pp. 152-184.

Santanach i Suñol, J. (2020), «L'incunable fantasma del Llibre del coc de mestre Robert (Toledo, 1477)», Llengua i literatura, 30, pp. 85-96).

Scappi, B. (2007 [1570]) Opera dell'arte del cucinare, Sala Bolognese, Forni Editore. Ed. facsímil.

Toury, G. (2004), Los estudios descriptivos y más allá. Metodología de la investigación en los estudios de traducción, Madrid, Cátedra.

Val, J. del (1971) Introducción» a D. Granado, Libro del Arte de Cocina, Madrid, Sociedad de bibliófilos españoles, pp. IX-XLVI.

SCRIPTA, Revista internacional de literatura i cultura medieval i moderna, núm. 18 / desembre 2021 / pp. 211-222 ISSN: 2340-4841 · doi:10.7203/SCRIPTA.18.22776 